TAUP 2134-94

\title{
The Unstable System \\ in \\ Relativistic Quantum Mechanics
}

\author{
L.P. Horwitz * \\ School of Physics \\ Raymond and Beverly Sackler Faculty of Exact Sciences \\ Tel Aviv University, Ramat Aviv, Israel
}

\begin{abstract}
A soluble model for the relativistically covariant description of an unstable system is given in terms of relativistic quantum field theory, with a structure similar to Van Hove's generalization of the Lee model in the non-relativistic theory. Since the Fock space for this model can be decomposed to sectors, it can be embedded in a one-particle Hilbert space in a spectral form similar to the Friedrichs model in the non-relativistic theory. Several types of spectral models result corresponding to physically motivated assumptions made in the framework of the field theory. For example, the continuous spectrum of the unperturbed problem may be $(-\infty, \infty)$ or semibounded. In the decay $V \rightarrow N+\theta$, the $\theta$ may have negative energy. In this case, the reaction corresponds to the crossed channel process $V+\bar{\theta} \rightarrow N$.
\end{abstract}

* Also at Department of Physics, Bar Ilan University, Ramat Gan, Israel 


\section{Introduction}

The Lee-Friedrichs model ${ }^{1,2}$ has been very useful for the study of the properties of unstable systems. It is completely soluble, and provides a framework for the analytic study of decay and scattering systems ${ }^{3}$. The construction of generalized states with exact exponential decay ${ }^{4}$ in the rigged Hilbert space $^{5}$ (Gel'fand triple) was originally motivated by the properties of this model; such states have found application in the theory of iterated maps $^{6}$ and play an important role in the study of irreversible processes ${ }^{7}$. For both theoretical development and physical applications, it is important to have a relativistic model for unstable systems. An unstable particle generally decays into a final state of two or more particles in a process for which the total energy is conserved, but the total mass is not. The equivalence between mass and energy, which enters quantitatively in the kinematical description of such a process, is a fundamentally relativistic relation. We shall use a formulation of relativistic quantum theory (and quantum field theory, as will be discussed in Section 2) which is well-suited for this problem in that it treats the energy and momentum of a particle as independent observables; hence, the mass becomes a dynamical variable. The transition from the undecayed initial state to the decayed system in the final state can then be studied as a continuous quantum mechanical evolution.

This formulation ${ }^{8}$, a generalization to the $N$-body system of the relativistic quantum theory of Stueckelberg ${ }^{9}$ developed for a single particle, describes evolution with a Poincaré invariant parameter $\tau$, sometimes called the "historical time." This invariant parameter corresponds to the universal time of (freely falling) ideal clocks ${ }^{10}$, and may be identified with the universal time originally postulated by Newton. In this quantum theory, the four momenta $p^{\mu}$ are conjugate to the four coordinates of space and time $x^{\mu}=(t, \mathbf{x})$. Classically, $t$, the time at which an event occurs in the laboratory (measured on a standard ideal clock, i.e., running with $\tau$ ), is a dynamical variable on the same footing as $\mathbf{x}$ (the place at which the event occurs in the laboratory). The wave function, for example, for a oneparticle system, $\psi_{\tau}(x)$ (we shall use $x$ to represent $x^{\mu}$ ), is the amplitude for the probability density $\left|\psi_{\tau}(x)\right|^{2}$ to find an event at $(t, \mathbf{x})$ at the historical time $\tau$. Since this density is

normalized with respect to integration over $t$, $\mathbf{x}$ (i.e., it belongs to the Hilbert space $L^{2}\left(\mathbf{R}^{4}\right)$ ) measurable unbounded excursions in $t$, as for $\mathbf{x}$ in the non-relativistic quantum theory, are not admissible ${ }^{11}$.

Classically, the variables $t(\tau), \mathbf{x}(\tau)$, correspond to the value of $t, \mathbf{x}$ of a signal detected in the laboratory (in some local inertial Lorentz frame) for an event generated at time $\tau$ (in some, perhaps other, frame). The result of the detection experiment is influenced by forces through the Hamilton equations $(i=1,2, \ldots, N$ for an $N$-body system)

$$
\frac{d x_{i}^{\mu}}{d \tau}=\frac{\partial K}{\partial p_{i \mu}} \quad \frac{d p_{i}^{\mu}}{d \tau}=-\frac{\partial K}{\partial x_{i \mu}}
$$

where $K$ is a function on the $8 N$ dimensional phase space, as well as the state of motion of the frame by means of the Lorentz transformation.

In the quantum theory, the Stueckelberg-Schrödinger equation for an $N$-body system

$$
i \frac{\partial \Psi_{\tau}}{\partial \tau}\left(x_{1} \ldots x_{N}\right)=K\left(x_{1} \ldots x_{N}, p_{1} \ldots p_{N}\right) \Psi_{\tau}\left(x_{1} \ldots x_{N}\right)
$$


describes the evolution of the wave function, and the dynamical variables satisfy the Heisenberg equations

$$
\dot{x}^{\mu} \equiv \frac{d x^{\mu}}{d \tau}=i\left[K, x^{\mu}\right] \quad \dot{p}^{\mu} \equiv \frac{d p^{\mu}}{d \tau}=i\left[K, p^{\mu}\right]
$$

with $\left[g^{\mu \nu}=(-,+,+,+)\right.$; we shall use units for which $\left.\hbar=c=1\right]$

$$
\left[x^{\mu}, p^{\nu}\right]=i g^{\mu \nu}
$$

For a single free particle, the assumption

$$
K_{0}=\frac{p^{\mu} p_{\mu}}{2 M}
$$

where $M$ is an intrinsic parameter belonging to the particle which sets the scale of energymomentum relative to its space-time evolution in $\tau$, results in (both classically and quantum mechanically)

$$
\frac{d x^{\mu}}{d \tau}=\frac{p^{\mu}}{M}
$$

For the classical case, one may eliminate $d \tau$ to obtain

$$
\frac{d \mathbf{x}}{d t}=\frac{\mathbf{p}}{E}
$$

the Einstein relation for velocity. A localized ( in $\mathbf{x}, t$ ) free wave packet moves with $\tau$, according to the Ehrenfest theorem, approximately along the classical world line. The generic form (one may also include electromagnetic interactions in gauge invariant form)

$$
K=\sum_{i=1}^{N} \frac{p_{i}^{\mu} p_{i \mu}}{2 M_{i}}+V\left(x_{1} \ldots x_{N}\right)
$$

where $V$ is a Poincaré invariant function of the $\left\{x_{i}^{\mu}\right\}$, provides a family of consistent direct action potential models to describe the evolution of a relativistic $N$-body system. The Gibbs ensembles for the equilibrium relativistic statistical mechanics of such systems, both for distinguishable and indistinguishable particles has been worked out ${ }^{12}$, as well as the BBGKY and Boltzmann equations ${ }^{13}$ for the non-equilibrium evolution of the $N$ body system. The equilibrium limit of the Boltzmann distribution provides a relativistic equilibrium mass distribution, for which the low energy and non-relativistic limits have been investigated (with results, in this limit, of course, in agreement with non-relativistic statistical mechanics), and the relativistic Fermi and Bose equilibrium distributions are currently being studied, for example, for their consequences in astrophysics and condensed matter theory ${ }^{14,15}$. Van Hove ${ }^{16}$ has used the non-relativistic Lee-Friedrichs model to discuss some of the fundamental properties of non-equilibrium statistical mechanics, and we shall, in a future publication, apply the model that we have developed here to analogous considerations. 
As an illustration of the applicability of the model (1.8), the two-body problem with "central" potential has been studied in detail ${ }^{17}$. Since we shall make use of two body kinematics in the study of the decay problem, we shall review here some of the details of the treatment of this problem. For the two-body case, we take

$$
K=\frac{p_{1}^{\mu} p_{1 \mu}}{2 M_{1}}+\frac{p_{2}^{\mu} p_{2 \mu}}{2 M_{2}}+V(\rho)
$$

where $\rho=\sqrt{x^{\mu} x_{\mu}}$, and the relative space-time coordinates are given obey

$$
x^{\mu}=x_{1}^{\mu}-x_{2}^{\mu} .
$$

In the non-relativistic limit, $t_{1} \rightarrow t_{2}$, so that $\rho \rightarrow r=\left|\mathbf{x}_{1}-\mathbf{x}_{2}\right|$. The total momentum of the system is

$$
P^{\mu}=p_{1}^{\mu}+p_{2}^{\mu}
$$

which commutes with the relative coordinate $x^{\mu}$. The operator $K$, as for the nonrelativistic analog, can therefore be written as

$$
K=\frac{P^{\mu} P_{\mu}}{2 M}+K_{r e l}
$$

where

$$
\begin{aligned}
K_{\text {rel }} & =\frac{p^{\mu} p_{\mu}}{2 m}+V(\rho), \\
p^{\mu} & =\frac{M_{2} p_{1}^{\mu}-M_{1} p_{2}^{\mu}}{M_{1}+M_{2}},
\end{aligned}
$$

corresponds to the evolution operator for the relative motion. Clearly, $K$ has only continuous spectrum, since the center of momentum motion is that of a free particle. Defining

$$
X^{\mu}=\frac{M_{1} x_{1}^{\mu}+M_{2} x_{2}^{\mu}}{M},
$$

where

$$
M=M_{1}+M_{2}
$$

we see that by $(1.1)$,

$$
\frac{d X^{\mu}}{d \tau}=\frac{P^{\mu}}{M}
$$

However, $K_{r e l}$, of (1.13), can have discrete or continuous spectrum in spite of the fact that $p^{\mu} p_{\mu}$ is a hyperbolic operator. One studies this problem by separating the center of momentum variable from $K$ (i.e., taking a direct integral representation of the solution over $P^{\mu}$, the absolutely conserved total energy-momentum). It is a remarkable fact that if we take the support of the eigenfunction $\psi_{k}(x)$, of the equation

$$
K_{r e l} \psi_{k}(x)=k \psi_{k}(x)
$$


to lie in the sub-measure space defined by

$$
x_{1}^{2}+x_{2}^{2}-t^{2} \geq 0,
$$

called the RMS (restricted Minkowski space), the spectrum of $K_{r e l}$ then coincides with the spectrum of the non-relativistic Schrödinger equation for all central potentials $V(r)=$ $\left.V(\rho)\right|_{\rho=r}$. The corresponding energies are then determined by (1.12), i.e., in the center of momentum frame, for which $\mathbf{P}=0$,

$$
E=\sqrt{2 M(k-K)} .
$$

With the assumption that the particles have masses close to $M_{1}, M_{2}$ when they are asymptotically spacelike separated (for which $V \approx 0$ ), and that the corresponding evaluation of $K$ is constant over the entire spectrum of $K_{r e l}$ [we argue that $t$-dependent perturbations of $V$ at long intervals can shift the spectrum of $K_{\text {rel }}$, but leave the absolutely conserved $K$ constant], (1.19) becomes

$$
E \cong \sqrt{2 M k+M^{2}} .
$$

For the case of discrete spectrum, where $k=k_{n}, n$ integer, when the excitations are small in magnitude compared to $M$,

$$
E_{n} \cong M+k_{n}+M O\left(\left(\frac{k_{n}}{M}\right)^{2}\right) .
$$

The same decompostion can be carried out for continuum values of $k$ (scattering states) small compared to $M$.

The RMS has a natural coordinatization in terms of the compact parameters $\theta, \phi$ and the non-compact parameter $\beta$ (along with $\rho$ ) as

$$
\begin{aligned}
x_{1} & =\rho \cos \phi \sin \theta \cosh \beta \\
x_{2} & =\rho \sin \phi \sin \theta \cosh \beta \\
x^{0}=t & =\rho \sin \theta \sinh \beta \\
x_{3} & =\rho \cos \theta ;
\end{aligned}
$$

clearly, $x_{1}^{2}+x_{2}^{2}-t^{2}=\rho^{2} \sin ^{2} \theta \geq 0$, and $x_{1}^{2}+x_{2}^{2}+x_{3}^{2}-t^{2}=\rho^{2} \geq 0$ as well. This submeasure space is $O(2,1)$ invariant, but not $O(3,1)$ invariant. The representations of $O(3,1)$ are induced from the irreducible representations of $O(2,1)$ which are provided by the eigenfunctions of $(1.17)^{17}$.

A model for relativistic hydrogen is obtained by taking $V=-e^{2} / \rho$. The exact eigenfunctions are therefore known for this problem; it has recently been shown that the selection rules for radiation coincide with those predicted by the non-relativistic theory ${ }^{18}$, and a relativistic form of the Zeeman effect has been worked out ${ }^{19}$. Many interesting group theoretical problems have been posed by this structure. The dynamical group for the relativistic Kepler problem has been found ${ }^{20}$, and a general investigation of such non-compact dynamical groups is being carried out ${ }^{21}$. 
The theory of unstable systems that forms the basis of our discussion in this work was first given in a systematic way by Wigner and Weisskopf ${ }^{22}$, in their work on the spectral line width for atomic radiation. In this (non-relativistic) theory, the unstable system is represented by a wave function $\psi$ which is usually taken to be an eigenfunction of an unperturbed Hamiltonian $H_{0}$; the action of the full perturbed Hamiltonian $H$ then causes a non-trivial evolution of the wave function $\psi_{t}=e^{-i H t} \psi$, and the amplitude

$$
A(t)=\left(\psi, e^{-i H t} \psi\right)
$$

is understood to be the amplitude for survival of the initial state, i.e.,

$$
p(t)=|A(t)|^{2}
$$

is the probability to find the initial state "undecayed" after a time $t$. After a time $t$, not too short and not too long, the evolution (1.23) is, for a wide class of models, well-approximated by the exponential of a complex number, i.e., of the form $\exp \left\{-i\left(E_{0}-i \frac{\gamma}{2}\right) t\right\}$, where $\gamma$ is the exponential decay rate for $p(t)$. If $H$ is defined on $\psi$, it is well known that the survival probability $p(t)$ has zero derivative at $t=0$, and, moreover, it goes asymptotically, for large $t$, as an inverse power ${ }^{23}$ of $t$.

It should be remarked in this connection that a fundamental assumption underlying the Wigner-Weisskopf theory is that, under continuous evolution,

$$
e^{-i H t} \psi=A(t) \psi+\psi^{\perp}
$$

where $\psi^{\perp}$ is the part of $e^{-i H t} \psi$ orthogonal to $\psi$. The linear superposition $A(t) \psi+\psi^{\perp}$ is, in fact, not seen in the laboratory. One generally sees only the state $\psi$ or the decay products. It appears that the time of decay is a superselection rule, and that this linear superposition does not correspond to the physical situation. This problem has been discussed by Horwitz and Piron ${ }^{24}$, where an alternative formulation of the problem for the non-relativistic case has been given. We shall not discuss the relativistic generalization of that treatment here, but confine ourselves to the usual Wigner-Weisskopf form which, although perhaps not the most general theoretical framework, has led to a good quantitative approximation for the non-relativistic theory.

One may understand the time evolution predicted by the Wigner- Weisskopf theory most easily by examining the inverse Laplace transform ${ }^{25}$ (one takes $A(t)=0$ for $t<0$ )

$$
A(t)=\frac{1}{2 \pi i} \int_{C} d z\left(\psi, \frac{1}{z-H} \psi\right) e^{-i z t}
$$

where $C$ is a contour that depends on the nature of the spectrum of $H$.

If $H$ has continuous spectrum, for example, on $(0, \infty)$, then the function

$$
R(z)=\left(\psi, \frac{1}{z-H} \psi\right)
$$

is analytic in the upper half plane along with its extension in the first Riemann sheet to the lower half plane, excluding the cut along the positive real axis. The contour $C$ then 
corresponds to a line running above the positive real axis from $\infty$ to 0 , then going around the branch point and continuing from 0 to $\infty$ below the real axis. The lower part of this contour can be deformed to a vertical line from 0 to $-i \infty$ for $t>0$, providing only a small contribution for large $t$. The part of the contour lying above the real axis may then be deformed through the cut to the second Riemann sheet, lowering it eventually to a line in the second sheet from $-i \infty$ to 0 , going around the branch point to connect with the part of contour that remains in the first sheet. In the course of this deformation, the contour passes the pole which has been shifted (for small coupling) from the discrete (bound) initial state embedded in the continuum on the real axis into the second sheet in the lower half plane, and this pole and its residue, proportional to $e^{-i \zeta t}$, where $\zeta$ is the position of the pole, corresponds to the exponential decay law. For large $t$, this contribution becomes small compared to that provided by the neighborhood of the branch point, which yields an inverse power of $t$. For very short times, the contribution of the deformed integral cannot be ignored, and, in fact, the integral is best approximated along the real axis ${ }^{26}$. Since $H$ is self-adjoint, clearly $d p(t) / d t$ vanishes at $t=0$, and it is easily seen that the power series in $t$ starts as $1-O\left(t^{2}\right)$.

Friedrichs ${ }^{2}$ proposed the following soluble model, which provides a closed analytic form for what is called the reduced resolvent $R(z)$, for which the analysis described above may be carried out in a very simply. Let

$$
H=H_{0}+V
$$

where $H_{0}$ has an absolutely continuous spectrum in $(0, \infty)$ and a single bound (discrete) state $\psi$ for which

$$
H_{0} \psi=E_{0} \psi
$$

Furthermore, suppose that on the continuous spectrum of $H_{0}$ characterized by

$$
\left.\left\langle E\left|H_{0}\right| f\right)=E\langle E| f\right)
$$

for any $f$ in the domain of $H_{0}$, and that

$$
\left\langle E|V| E^{\prime}\right\rangle=0
$$

for all $E, E^{\prime}$. The diagonal part $(\psi|V| \psi)$ of $V$, leading to a simple shift in $E_{0}$, is usually taken to be zero. Then, it follows from the identity (second resolvent equation)

$$
\frac{1}{z-H}=\frac{1}{z-H_{0}}+\frac{1}{z-H_{0}} V \frac{1}{z-H}
$$

that

$$
R(z)=\frac{1}{z-E_{0}}+\frac{1}{z-E_{0}} \int d E(\psi|V| E\rangle\left\langle E\left|\frac{1}{z-H}\right| \psi\right)
$$

and

$$
\left.\left\langle E\left|\frac{1}{z-H}\right| \psi\right\rangle=\frac{1}{z-E}\langle E| V \psi\right) R(z)
$$


The simplifying truncation in (1.33) follows from the essential feature (1.30) of the model, implying physically that there are no "final state" interactions. This is often, in fact, a good physical approximation in decaying systems. Substituting (1.33) into (1.32), one finds

$$
h(z) R(z)=1,
$$

where

$$
h(z)=z-E_{0}-\int d E \frac{|\langle E|V| \psi)|^{2}}{z-E} .
$$

The singularities of $R(z)$ arise from the vanishing of the function $h(z)$. Since

$$
\operatorname{Im} H(z)=\operatorname{Im} z\left[1+\int d E \frac{|\langle E|V| \psi)|^{2}}{|z-E|^{2}}\right],
$$

there can be no zero in the first sheet of the function for $\operatorname{Im} z \neq 0$. For sufficiently small $V$, it is easy to show that there is no zero on the negative real axis. Passing to the second sheet continuously through the cut, one finds ${ }^{25}$

$$
h^{I I}(z)=h(z)+2 \pi i W(z),
$$

where $W(z)$ is the analytic extension of

$$
W(E)=|\langle E|V| \psi)|^{2}
$$

to a region of the lower half plane sufficiently large [that is, we assume an analytic function in this domain whose boundary is $W(E)]$ to include the possible zero of $h^{I I}(z)$. If this zero occurs for $z=\zeta$ with small imaginary part, then

$$
\operatorname{Im} \zeta\left[1+\int d E \frac{|\langle E|V| \psi)|^{2}}{|z-E|^{2}}\right] \cong-2 \pi \mid\left\langle E_{0}|V| \psi||^{2},\right.
$$

so that, recalling the representation

$$
\lim _{\epsilon \rightarrow 0} \frac{1}{\pi} \frac{\epsilon}{\epsilon^{2}+x^{2}}=\delta(x),
$$

one sees that the pole occurs at

$$
\operatorname{Im} \zeta \cong-\pi\left|\left\langle E_{0}|V| \psi\right)\right|^{2} .
$$

It is clear that for the description of, for example, particle decay, involving a real change in the the total particle mass of the system, a relativistic theory is required. Since, as pointed out by Henley and Thirring ${ }^{27}$, in the standard on-shell relativistic quantum field theory, both the annihilation operator for a particle and the creation operator for an antiparticle occur linearly combined in the local field operator, a relativistic analog of the model described above (in the framework of quantum field theory, in the form given by 
Lee $^{1}$ ) would contain, along with the process $n \leftrightarrow p+\pi^{-}$, automatically and simultaneously, the process $p \leftrightarrow n+\pi^{+}$. Zumino ${ }^{28}$ has remarked that the expression $E=\sqrt{\mathbf{p}^{2}+m^{2}}$ is not analytic, and creates singularities that make the analytic continuations we have discussed above difficult; he suggested, toward the end of his article, the use of the quadratic form $p^{\mu} p_{\mu}$ instead, a form which we will, in fact, utilize in the formulation we give in the next sections. In the field theoretic form, the "coupling" which plays the role of $\langle E|V| \psi)$, is a function of the momentum of one of the particles emitted in the decay; its Fourier transform to configuration space results in a non-local function in space which would make Lorentz invariance difficult to achieve. The original form of the model proposed by Lee ${ }^{1}$, which was constructed to achieve the simplest form, did not allow for recoil, and hence its relativistic generalization would destroy Poincaré invariance; however, in the form given by Van Hove ${ }^{29}$, recoil is taken into account. It is for this form of the field theory which we shall provide a relativistic generalization.

It is the purpose of this paper to show how such a relativistically covariant version of this model can be constructed. In Section 2, we shall formulate the decay problem in terms of the relativistic quantum field theory resulting from the second quantization of the covariant quantum theory we have discussed above. It has recently been shown that such a field theory results in a consistent quantum electrodynamics ${ }^{30}$ involving a five dimensional generalization of the Maxwell field. This field (including a Lorentz scalar field as the fifth component) reduces to the usual Maxwell field when a characteristic correlation length reaches a critical value. The theory has been shown ${ }^{31}$ to provide insight into regularization (such as that of Pauli-Villars ${ }^{32,33}$ ) and renormalization methods used in standard quantum electrodynamics (e.g., refs. 34). We shall not describe here this general theory, but use the relativistic matter fields of this theory in much the same way that Lee $^{1}$ and, in particular, Van Hove ${ }^{29}$ used the non-relativistic quantized fields to describe the soluble Lee model. The information we obtain from this field theory, in a model which has two conserved quantum numbers defining particle number sectors, is then used, in Section 4, to construct a corresponding model in a single Hilbert space (which is not a tensor product Fock space representing a quantum field theory) according to the method of Friedrichs ${ }^{2}$. We thus achieve a relativistically covariant Lee-Friedrichs soluble model for an unstable system.

It is a pleasure to dedicate this paper to Professor Jean-Pierre Vigier on the $45^{\text {th }}$ anniversary of his joining Louis de Broglie at the Institut Henri Poincaré. The theory of E.C.G. Stueckelberg provides a framework in which the notion of de Broglie ${ }^{35}$, that the mass of a particle is associated with a quantum frequency, is realized through the identification of the energy in a relativistic theory as an observable kinematically independent of the momentum. The work of Vigier and collaborators (ref. 8), contributing to the development of this theory, we feel, makes the present work appropriate for such an occasion.

\section{Field Theory Model}

In order to determine the structure and physically motivated basic assumptions to be imposed on a Friedrichs type model for the relativistic description of an unstable system in a "one- particle" Hilbert space, we first study in some detail a model in relativistic quantum 
field theory. We shall assume boson matter fields in what follows. The properties of the quantum fields may be obtained by a variety of methods; one of these is the construction of a Lagrangian density for which the variation of the associated action yields a field equation which coincides with the Stueckelberg-Schrödinger equation. A Lagrangian density which yields this result is, for example ${ }^{30}$,

$$
\begin{aligned}
\mathcal{L} & =\frac{1}{2}\left\{i \psi_{\tau}^{\dagger} \partial_{\tau} \psi_{\tau}-\partial_{\mu} \psi_{\tau}^{\dagger} \partial^{\mu} \psi_{\tau}+\text { c.c. }\right\} \\
& \left.-\int d x d x^{\prime} \psi_{\tau}^{\dagger}(x) \psi_{\tau}^{\dagger}\left(x^{\prime}\right) V\left(x-x^{\prime}\right) \psi_{\tau}\left(x^{\prime}\right) \psi_{\tau}(x)\right\}
\end{aligned}
$$

and the action is defined as

$$
S=\int d \tau \int d^{4} x \mathcal{L}
$$

It then follows that the field conjugate to $\psi$ is $\Pi \equiv i \psi^{\dagger}$, so that the equal- $\tau$ commutation relations are

$$
\left[\psi_{\tau}(x), \psi_{\tau}^{\dagger}\left(x^{\prime}\right)\right]=\delta^{4}\left(x-x^{\prime}\right)
$$

In momentum space, for which

$$
\psi_{\tau}(p)=\frac{1}{(2 \pi)^{2}} \int d^{4} x e^{-i p^{\mu} x_{\mu}} \psi_{\tau}(x),
$$

this relation becomes

$$
\left[\psi_{\tau}(p), \Pi_{\tau}\left(p^{\prime}\right)\right]=i \delta^{4}\left(p-p^{\prime}\right) .
$$

To see how this relation goes over to the on-mass-shell field theory, we follow a nonrigorous but instructive procedure. Multiply both sides of (2.5) by $\triangle E$, and notice that for $E=\sqrt{\mathbf{p}^{2}+m^{2}}$, where $m^{2}$ is a dynamical variable, $\triangle E=\triangle m^{2} / 2 E$, we obtain

$$
\left[\tilde{\psi}_{\tau}(\mathbf{p}), \tilde{\Pi}\left(p^{\prime}\right)\right]=2 i E \delta^{3}\left(\mathbf{p}-\mathbf{p}^{\prime}\right)
$$

where $\tilde{\psi}_{\tau}(\mathbf{p})=\sqrt{\triangle m^{2}} \psi_{\tau}(p)$, and $\tilde{\Pi}_{\tau}(\mathbf{p})=\sqrt{\triangle m^{2}} \Pi_{\tau}(p)$. We may then take the limit $\triangle m^{2} \rightarrow 0, m^{2} \rightarrow m_{0}^{2}$, a fixed constant (in this limit, $t \rightarrow \tau$ as well ${ }^{8}$ ). The operator-valued distribution $\psi_{\tau}(p)$ therefore peaks in support (in all matrix elements) sharply at $m^{2} \sim m_{0}^{2}$ in this limit. Eq. (2.6) is the conventional equal time commutation relation.

The example we have studied above assumes positive energy. Both fields $\psi, \Pi$ may have negative energy as well, but the right hand side vanishes if one has positive, and the other negative $\left(\triangle E \delta\left(E-E^{\prime}\right)\right.$ would be zero in this case). We remark, furthermore, that according to $(2.5)$, the field $\psi(x)$ contains only the annihilation operator $\psi_{\tau}(p)$, for both positive and negative energy, but not a creation operator for the antiparticle (as for the second quantization of the Schrödinger wave function). The CPT conjugate of a negative energy function corresponds to the representation of the antiparticle observed in the laboratory. This is consistent with Stueckelberg's (and Feynman's) ${ }^{9}$ original interpretation, associating the antiparticle with the particle going "backwards" (with $\tau$ ) in time. The quantum electrodynamics (a $U(1)$ gauge field in interaction with the matter field) 
associated with this theory is obtained by requiring gauge invariance of the Stueckelberg equation $^{30}$. In addition to the derivative $-i \partial^{\mu}$, which becomes the gauge covariant derivative $-i \partial^{\mu}-a^{\mu}(x, \tau)$, the derivative $i \partial_{\tau}$ also requires compensation, i.e., $i \partial_{\tau} \rightarrow i \partial_{\tau}+a_{5}(x, \tau)$, introducing a fifth, Lorentz scalar, field.

It has been shown that the Maxwell potential (satisfying the usual Maxwell equations with a four dimensional conserved current as its source) is obtained from the field $a^{\mu}(x, \tau)$ by integration over $\tau^{30}$, i.e., the Maxwell field is the zero mode of the "pre-Maxwell" field (the $a^{5}$ field decouples in the $U(1)$ theory). In the limit in which the Fourier transform $a^{\mu}(x, s)$ on $\tau$ of the pre-Maxwell fields have support only in a small interval $\triangle s$ near zero (a long correlation length limit) one can show that the theory goes over to the usual form of quantum electrodynamics ${ }^{30}$. The theory has been consistently quantized, using canonical methods $^{36}$, in full generality. In the quantized form, the theory goes over to the standard quantum electrodynamics, carrying Pauli-Villars regularization ${ }^{33,34}$, in the long correlation length limit, as we have remarked above. We shall not discuss this structure further here, but concentrate on a model field theory with a generalized invariant Hamiltonian of the form

$$
K=K_{0}+K_{I}
$$

where (we write $p^{2} \equiv p^{\mu} p_{\mu}, k^{2} \equiv k^{\mu} k_{\mu}$ henceforth)

$$
\begin{aligned}
K_{0} & =\int d^{4} p \frac{p^{2}}{2 M_{V}} b^{\dagger}(p) b(p) \\
& +\int d^{4} p \frac{p^{2}}{2 M_{N}} a_{N}^{\dagger}(p) a_{N}(p) \\
& +\int d^{4} k \frac{k^{2}}{2 M_{\theta}} a^{\dagger}(k)_{\theta} a_{\theta}(k),
\end{aligned}
$$

and

$$
\begin{aligned}
K_{I} & =\int d^{4} k d^{4} p\left\{f(k) b^{\dagger}(p) a_{N}(p-k) a_{\theta}(k)\right. \\
& \left.+f(k)^{*} a_{N}^{\dagger}(p-k) a_{\theta}^{\dagger}(k) b(p)\right\},
\end{aligned}
$$

describing the process $V \leftrightarrow N+\theta$. Here, $b(p)$ is the annihilation operator for the $V$ particle, and $a_{N}(p), a_{\theta}(k)$, for the $N$ and $\theta$ respectively. This model is a relativistic generalization of Van Hove's form ${ }^{29}$ of the Lee model for the non-relativistic theory (note that expressing the "kinetic energy" in the on-mass-shell form $\sqrt{\mathbf{p}^{2}+m_{0}^{2}}$ would make it impossible to separate the center of momentum motion in the two-body channel). The operators

$$
\begin{aligned}
Q_{1} & =\int d^{4} p\left[b^{\dagger}(p) b(p)+a_{N}^{\dagger}(p) a_{N}(p)\right] \\
Q_{2} & =\int d^{4} p\left[a_{N}^{\dagger}(p) a_{N}(p)-a_{\theta}^{\dagger}(p) a_{\theta}(p)\right]
\end{aligned}
$$

are conserved (as one easily sees using the commutation relations (2.5)), and enable us to decompose the Fock space to sectors. We shall study this problem in the lowest sector $Q_{1}=1, Q_{2}=0$ for which there is just one $V$ or one $N$ and one $\theta$. 
We now wish to calculate

$$
\Psi_{\tau}=e^{-i K \tau} \Psi_{0},
$$

where we take the normalized wave packet

$$
\Psi_{0}=\int g(p) b^{\dagger}(p) d^{4} p|0\rangle
$$

as the one-particle initial state (the $V$-particle). We can now compute the relativistic analog of the survival amplitude

$$
A_{s}(\tau)=\left(\Psi_{0}, \Psi_{\tau}\right)=\left(\Psi_{0}, e^{-i K \tau} \Psi_{0}\right)
$$

as a function of the invariant time $\tau$. The initial state may be chosen so that $\tau$ is the mean proper time for the undecayed system (i.e., for which $\left\langle\left(d x^{\mu} / d \tau\right)\right\rangle\left\langle\left(d x_{\mu} / d \tau\right)\right\rangle=-1$ ).

Since $A(\tau)$ is Lorentz invariant, we may transform from an arbitrary frame to the (average) rest frame of the initial state under the evolution induced by $K$. Its $\tau$-dependence therefore clearly can arise only from the evolution of $\Psi_{0}$ to a different channel (assumed here to be more rapid than the significant spreading of the wave packet).

In the sector $Q_{1}=1$ and $Q_{2}=0$, conserved by the evolution, a general representation for the Fock space state $\Psi_{\tau}$ is

$$
\Psi_{\tau}=\int d^{4} p A(p, \tau) b^{\dagger}(p)|0\rangle+\int d^{4} p d^{4} k B(p, k, \tau) a_{N}^{\dagger}(p) a_{\theta}^{\dagger}(k)|0\rangle,
$$

where we choose for the initial condition

$$
\begin{aligned}
A(p, 0) & =g(p) \\
B(p, k, 0) & =0,
\end{aligned}
$$

so that the initial state is that given by (2.12). The evolution of the system is given by

$$
i \frac{\partial \Psi_{\tau}}{\partial \tau}=K \Psi_{\tau},
$$

the quantum field theory form of Eq. (1.2). Applying the definitions (2.8), (2.9) for $K$ and the commutation relations for the annihilation-creation operators, one obtains the differential equations

$$
i \frac{\partial A}{\partial \tau}(p, \tau)=\frac{p^{2}}{2 M_{V}} A(p, \tau)+\int d^{4} k f(k) B(p-k, k, \tau)
$$

and

$$
i \frac{\partial B}{\partial \tau}(p, k, \tau)=\left(\frac{p^{2}}{2 M_{N}}+\frac{k^{2}}{2 M_{\theta}}\right) B(p, k, \tau)+f^{*}(k) A(p+k, \tau) .
$$


Defining the Laplace transforms

$$
\begin{aligned}
\tilde{A}(p, z) & =\int_{0}^{\infty} e^{i z \tau} A(p, \tau) d \tau \\
\tilde{B}(p, k, z) & =\int_{0}^{\infty} e^{i z \tau} B(p, k, \tau),
\end{aligned}
$$

we obtain from $(2.17),(2.18)$ the relations

$$
\left(z-\frac{p^{2}}{2 M_{V}}\right) \tilde{A}(p, z)=i g(p)+\int d^{4} k f(k) \tilde{B}(p-k, k, z),
$$

where the term $i g(p)$ arises from the boundary point at $\tau=0$ of the integration by parts (the contribution at $\tau \rightarrow \infty$ vanishes for $z$ in the upper half plane), and

$$
z \tilde{B}(p, k, z)=\left(\frac{p^{2}}{2 M_{N}}+\frac{k^{2}}{2 M_{\theta}}\right) \tilde{B}(p, k, z)+f^{*}(k) \tilde{A}(p+k, z) .
$$

Substituting (2.21) into (2.20), we obtain the covariant analog of Eq. (1.34),

$$
h(p, z) \tilde{A}(p, z)=i g(p)
$$

where

$$
h(p, z)=z-\frac{p^{2}}{2 M_{V}}-\int d^{4} k \frac{|f(k)|^{2}}{z-\frac{(p-k)^{2}}{2 M_{N}}-\frac{k^{2}}{2 M_{\theta}}} .
$$

In this form, one recognizes the essential content of the relativistic Lee model. The term $p^{2} / 2 M_{V}$ corresponds to the mass (squared) of the unstable system, i.e., the discrete (for fixed $p^{\mu}$ ) eigenvalue of the unperturbed initial state, and the cut, the singularity corresponding to the continuous spectrum of the decay channel.

The Laplace transform of the survival amplitude (2.13),

$$
\tilde{A}_{s}(z)=\int_{0}^{\infty} e^{i z \tau} A_{s}(\tau) d \tau=i\left(\Psi_{0}, \frac{1}{z-K} \Psi_{0}\right)
$$

analytic for $z$ in the upper half-plane, is then given, according to (2.12) and (2.14), by

$$
\tilde{A}_{s}(z)=\int d^{4} p g(p)^{*} \tilde{A}(p, z)
$$

i.e.(with $(2.22))$,

$$
\tilde{A}_{s}(z)=i \int d^{4} p \frac{|g(p)|^{2}}{h(p, z)}
$$

The inverse transform is

$$
A_{s}(\tau)=\frac{1}{2 \pi i} \int_{C} e^{-i z \tau} \tilde{A}_{s}(z) d z
$$


where $C$ is a contour taken from $+\infty+i \epsilon$ to $-\infty+i \epsilon$, i.e., just above the real axis. In the case of semi-bounded spectrum for the continuum channel, for $\tau>0$, the left hand part of the contour can be deformed to the lower half-plane, where this part of the contour (except for the neighborhood of the branch point) provides a small background contribution for large $\tau$. In the same way as discussed in Section 1, the right hand half line can be rotated into the second sheet, and brought down to the line $(0, \infty)$, picking up the contribution of the pole of $h(p, z)$ on the way. If the spectrum is unbounded, analytic continuation can be made by bringing the whole line of integration down into the lower half plane.

Note that the contribution of the pole corresponding to the zero of the analytic continuation of $h(p, z)$ is spread out by the integration (2.26), but this spreading can be very small (it occurs primarily in the real part of the pole, since the imaginary part, for weak coupling, is almost independent of $p$ ). In the next section, we discuss the structure of the spectrum of the continuous channel.

\section{The Spectrum}

Clearly, the unperturbed spectrum (for $f(k)=0$ ) for the relativistic Lee model, according to (2.8), is purely continuous. We see, however, from the structure of (2.23) that for each value of $p^{2}$, there is an effective discrete eigenstate with eigenvalue $p^{2} / 2 M_{V}$, considering the Hilbert space as a direct sum space over the absolutely conserved total energy-momentum, which coincides with $p^{\mu}$, of the system. The remaining terms in (2.8) can be written as

$$
\int d^{4} k d^{4} p\left\{\frac{(p-k)^{2}}{2 M_{N}}+\frac{k^{2}}{2 M_{\theta}}\right\} a_{N}^{\dagger}(p-k) a_{\theta}^{\dagger}(k) a_{\theta}(k) a_{N}(p-k),
$$

since we can replace $p-k$ by $p$; the integration of the first term over $k$ then provides a factor which is the number operator of the $\theta$ field, and the second term has a factor, after integration over $p$, which is the number operator for the $N$ field. In the sector $Q_{1}=1$ and $Q_{2}=0$, these factors are unity in the continuum channel and zero in the initial state channel, and hence do not change the valuation of this contribution to the unperturbed spectrum. Clearly, (3.1) corresponds to the continuous spectrum associated with the cut in $(2.23)$, for each fixed $p^{\mu}$. We therefore study the range of the function

$$
w(k)=\frac{(p-k)^{2}}{2 M_{N}}+\frac{k^{2}}{2 M_{\theta}} .
$$

The range of the contribution of the continuous spectrum to $h(p, z)$ in $(2.23)$ is determined by the support properties of the coupling function $f(k)$; it may contain, in an invariant way, restriction to $k^{0}$ positive or negative, or bounds on $m_{\theta}^{2} \equiv-k^{\mu} k_{\mu}$, the masssquared of the $\theta$ particle.

To study these cases, we write (3.2) as

$$
w(k)=\frac{p^{2}}{2 M_{N}}-\frac{m_{\theta}^{2}}{2}\left(\frac{1}{M_{\theta}}+\frac{1}{M_{N}}\right)+\frac{1}{M_{N}}\left( \pm p^{0} \sqrt{\mathbf{k}^{2}+m_{\theta}^{2}}-\mathbf{p} \cdot \mathbf{k}\right),
$$


where the signs \pm in (3.3) correspond to the choice of sign of the energy variable of the $\theta$ particle, i.e.,

$$
k^{0}= \pm \sqrt{\mathbf{k}^{2}+m_{\theta}^{2}}
$$

The choice of sign determines the direction of propagation of the wave packet of the $\theta$ particle in time. As pointed out by Stueckelberg (and Feynman) ${ }^{9}$, the negative energy particle has a correspondence with the antiparticle; the $C P T$ conjugate of the wave packet is that of a particle with positive energy and the opposite sign of the momentum and the charge, i.e., the antiparticle. Applying this definition to a particle with $\operatorname{spin}^{37}$, one finds that it is completely consistent with Dirac's interpretation (the representations of $C, P$ and $T$ on the corresponding Dirac spinors are the same as in the usual on-shell theory $)^{38}$. We first discuss the case $k^{0}>0$.

We shall take $p^{0}>0$ in the following; the $\theta$ and $N$ particles can exchange roles under a slight reformulation, and there is therefore a symmetry in the analysis for $p^{0}<0$. Then since $p^{0}>|\mathbf{p}|$, if the $V$ particle has positive mass-squared (this condition follows from the choice of states of the quantum field), it follows from (3.3) that

$$
w(k) \rightarrow+\infty \text { for }|\mathbf{k}| \rightarrow \infty
$$

The lower bound of the spectrum is determined, for each $p^{\mu}, m_{\theta}$, as a function of $|\mathbf{k}|$, by

$$
\frac{d w(k)}{d|\mathbf{k}|}=0
$$

Let us define the $\mathbf{k}$-dependent part of (3.3) as

$$
\eta_{+}(\mathbf{k})=p^{0} \sqrt{\mathbf{k}^{2}+m_{\theta}^{2}}-|\mathbf{p}||\mathbf{k}| \cos \phi
$$

where $\phi$ is the angle between $\mathbf{p}$ and $\mathbf{k}$. Then, this condition implies

$$
\eta_{+}^{\prime}(\mathbf{k})=\frac{p^{0}|\mathbf{k}|}{\sqrt{\mathbf{k}^{2}+m_{\theta}^{2}}}-|\mathbf{p}| \cos \phi .
$$

The minimum value is therefore (the second derivative is positive) achieved for $\cos \phi>0$, at

$$
p^{0^{2}} \mathbf{k}^{2}=\mathbf{p}^{2}\left(\mathbf{k}^{2}+m_{\theta}^{2}\right) \cos ^{2} \phi,
$$

yielding

$$
|\mathbf{k}|=\frac{|\mathbf{p}| \cos \phi}{\sqrt{p^{0^{2}}-\mathbf{p}^{2} \cos ^{2} \phi}} m_{\theta},
$$

so that

$$
\sqrt{\mathbf{k}^{2}+m_{\theta}^{2}}=\frac{m_{\theta} p^{0}}{\sqrt{p^{0^{2}}-\mathbf{p}^{2} \cos ^{2} \phi}}
$$


It then follows that

$$
\left(\eta_{+}(\mathbf{k})\right)_{\min }=m_{\theta} \sqrt{p^{0^{2}}-\mathbf{p}^{2} \cos ^{2} \phi},
$$

which is further minimized by taking $\phi=0$ :

$$
\left(\eta_{+}(\mathbf{k})\right)_{\min }=m_{\theta} \sqrt{-p^{2}}
$$

We note that for $\cos \phi<0$, the minimum value is at $p^{0} m_{\theta}$, always larger than the value given by (3.11) for $\mathbf{p} \neq 0$. The spectrum for the two-body $N+\theta$ channel is therefore

$$
w_{+}(k)=\left(\frac{p^{2}}{2 M_{N}}-\frac{m_{\theta}^{2}}{2}\left(\frac{1}{M_{\theta}}+\frac{1}{M_{N}}\right)+\frac{m_{\theta} \sqrt{-p^{2}}}{M_{N}}, \infty\right)
$$

Let us now return to $(3.3)$ for $k^{0}<0$. In this case,

$$
\eta_{-}(k)=-p^{0} \sqrt{\mathbf{k}^{2}+m_{\theta}^{2}}-\mathbf{p} \cdot \mathbf{k}
$$

goes to $-\infty$ for $|\mathbf{k}| \rightarrow \infty$, and the spectrum is unbounded from below. The upper bound for fixed $m_{\theta}$ occurs at a value of $|\mathbf{k}|$ for which (the second derivative is negative)

$$
\eta_{-}^{\prime}(k)=\frac{-p^{0}|\mathbf{k}|}{\sqrt{\mathbf{k}^{2}+m_{\theta}^{2}}}-|\mathbf{p}| \cos \phi
$$

yielding, for $\cos \phi<0$,

$$
|\mathbf{k}|=\frac{|\mathbf{p}||\cos \phi|}{\sqrt{p^{0^{2}}-\mathbf{p}^{2} \cos ^{2} \phi}} m_{\theta}
$$

so that

$$
\left(\eta_{-}(k)\right)_{\max }=-m_{\theta} \sqrt{p^{0^{2}}-\mathbf{p}^{2} \cos ^{2} \phi}
$$

which is further maximized by taking $\phi=\pi$ :

$$
\left(\eta_{-}(k)\right)_{\max }=-m_{\theta} \sqrt{-p^{2}}
$$

The spectrum for the two body $N+\theta$ channel in the case $k^{0}<0$ is then

$$
w_{-}(k)=\left(-\infty, \frac{p^{2}}{2 M_{N}}-\frac{m_{\theta}^{2}}{2}\left(\frac{1}{M_{\theta}}+\frac{1}{M_{N}}\right)-\frac{m_{\theta} \sqrt{-p^{2}}}{M_{N}}\right) .
$$

The upper bound of $w_{-}(k)$ is less than the lower bound of $w_{+}(k)$, i.e.,

$$
\left(w_{+}(k)\right)_{\min }-\left(w_{-}(k)\right)_{\max }=2 m_{\theta} \frac{\sqrt{-p^{2}}}{M_{N}},
$$


and there is a gap in the spectrum for every $m_{\theta} \neq 0$. For the case in which $m_{\theta}$ can reach zero, the continuous spectrum, including both $k^{0}>0$ and $k^{0}<0$ parts, covers the real line $(-\infty, \infty)$.

Let us consider the question, for $k^{0}>0$, of whether the "discrete" spectral point $p^{2} / 2 M_{V}$ is embedded in the continuum. The condition that this point lie above the lower bound of $w_{+}(k)$ is that

$$
\frac{m_{\theta}^{2}}{2}\left(\frac{1}{M_{N}}+\frac{1}{M_{\theta}}\right)-m_{\theta} \frac{\sqrt{-p^{2}}}{M_{N}}>\frac{p^{2}}{2}\left(\frac{1}{M_{N}}-\frac{1}{M_{V}}\right) .
$$

Although conservation of energy and momentum imply that for a decay process, $m_{V}>$ $m_{N}+m_{\theta}$, the dimensional scale factors $M_{V}, M_{N}, M_{\theta}$ may satisfy this inequality as well; when the kinematical masses $\sqrt{-p^{2}}, \sqrt{-(p-k)^{2}}$ and $\sqrt{-k^{2}}$ take on these values, the classical squared proper time intervals of the corresponding free world line $-d x^{\mu} d x_{\mu}$ become equal to $d \tau^{2}$; they may also be taken as the Galilean limit of the kinematical masse $^{12}$. In this case, $\frac{1}{M_{N}}-\frac{1}{M_{V}}$ is positive. The right hand side of (3.20) is therefore, in this case, negative. The minimum value of the left hand side, at

$$
\left(m_{\theta}\right)_{\min }=\frac{M_{\theta}}{M_{N}+M_{\theta}} \sqrt{-p^{2}}
$$

is

$$
-\frac{p^{2}}{2} \frac{M_{\theta}}{M_{N}\left(M_{N}+M_{\theta}\right)}>0,
$$

and hence, in this case the discrete spectral point is embedded. It is not, however, necessary to make the choice $M_{V}>M_{N}$; a choice of parameters can be made for which the discrete state lies below the minimum of $w_{+}(k)$. This would correspond to the isolated "bound state" case of the Lee model, for which some studies in mass renormalization have been made ${ }^{39}$.

As for the two body problem discussed in Eq. (1.9) and the following paragraph, the kinetic terms of the two body final state can be expressed in terms of the total momentum and relative momentum

$$
P^{\mu}=\left(p^{\mu}-k^{\mu}\right)+k^{\mu}=p^{\mu}
$$

and

$$
p_{r e l}^{\mu}=\frac{M_{\theta}\left(p^{\mu}-k^{\mu}\right)-M_{N} k^{\mu}}{M}=\frac{M_{\theta}}{M} p^{\mu}-k^{\mu}
$$

as

$$
w(k)=\frac{P^{2}}{2 M}+\frac{p_{r e l}^{2}}{2 m},
$$

where $M=M_{N}+M_{\theta}, \quad m=M_{N} M_{\theta} /\left(M_{N}+M_{\theta}\right)$.

For the scattering system corresponding to $N+\theta \rightarrow N+\theta$, the Lippmann-Schwinger equations provide an effective potential for transitions in $p_{\text {rel }}$, and the problem may be treated as discussed in Section 1. This will be done in detail elsewhere.

For the positive energy contributions, the process $V \rightarrow N+\theta$ is concerned only with particles in the usual sense, and may be represented as a process in space- time as in fig.1. 
[fig. 1]

For $k^{0}<0$, however, the space-time picture appears as in fig. 2, corresponding to Stueckelberg's representation of pair annihilation as part of the process $V \rightarrow N+\theta$.

[fig. 2]

The corresponding physical process, seen in the laboratory, contains the $C P T$ conjugate of the $\theta$ external leg, and appears as in fig. 3, corresponding to a real $V+\bar{\theta}$ annihilation to yield the (virtual) $N$.

[fig. 3]

The inverse process, $N \rightarrow V+\bar{\theta}$ corresponds to the physical decay of the $N$ particle. For the case $n \rightarrow p+\pi^{-}$discussed by Henley and Thirring ${ }^{27}$, this inverse process corresponds to $p \rightarrow n+\pi^{+}$. The difficulty that they cite, that in the on-shell relativistic quantum field theories both of these processes occur simultaneously in the interaction term of an analog relativistic Lee model, does not occur in the model presented here, since the two processes, although described in the context of the same theory, occur in two different parts of the spectrum.

For the case $k^{0}<0$, the condition that the discrete spectral point is embedded in the continuum is

$$
\frac{m_{\theta}^{2}}{2}\left(\frac{1}{M_{\theta}}+\frac{1}{M_{N}}\right)+m_{\theta} \frac{\sqrt{-p^{2}}}{M_{N}}<\frac{p^{2}}{2}\left(\frac{1}{M_{N}}-\frac{1}{M_{V}}\right) .
$$

If $M_{V}>M_{N}$, the right hand side is negative, and hence the discrete point is an isolated bound state. However, in the physical process $N \rightarrow V+\bar{\theta}$, the scale parameters may be chosen (as in the argument given for the process $V \rightarrow N+\theta$ ) to satisfy $M_{N}>M_{V}$, in which case the right hand side of (3.23) is positive. The left hand side can extend to zero, and hence for this choice, the discrete state is embedded in the continuum as well. Since, as we have seen, the inclusion of both positive and negative branches of $k^{0}$ results in a spectrum that covers the whole real line (when $m_{\theta}$ reaches zero), the discrete spectral point would then be embedded for any $M_{V}, M_{N}$.

\section{The Friedrichs Form and Conclusions}

Since the relativistic Lee model we have considered above has sectors, it can be isomorphically mapped to a spectral model. We write the generator of the motion in the direct integral space $\int_{\oplus} \mathcal{H}_{p}$ over the four-momenta of the $V$ particle as (for each $p^{\mu}$ )

$$
K_{p}=\mathcal{M}_{V}(p) P_{0}+\bar{K}_{p}+K_{I},
$$

where $K_{I}$ has matrix elements only between the continuum and the discrete state $\phi_{p}$ (here, $P_{0}=\phi_{p} \phi_{p}^{\dagger}$ ) with eigenvalue $\mathcal{M}_{V}(p)$ corresponding to $p^{2} / 2 M_{V}$, and $\bar{K}_{p}$ has absolute continuous spectrum (with, perhaps, finite or semi-infinite gaps). This form corresponds to the canonical decomposition

$$
K_{p}=P_{0} K_{p} P_{0}+\bar{P} K_{p} \bar{P}+P_{0} K_{p} \bar{P}+\bar{P} K_{p} P_{0},
$$

where $\bar{P}$ is the projection into the continuous spectrum (at each point $p$ ).

The vertex function $f(k)$ of the field theoretical model can be covariantly restricted to support in the upper light cone, $k^{0}>0$, and the spectrum (with bounded $m_{\theta}$ ) will be 
therefore similar to that of the usual non-relativistic Friedrichs model, i.e., for which the unperturbed evolution operator has semi-infinite absolutely continuous spectrum and an embedded discrete state with an eigenvalue corresponding to the mass of the initial state of the decay problem (note a similar phenomenon in structure of the Feynman propagators studied in ref. 40).

As we have seen, if $k^{0}$ can be positive or negative, the continuous spectrum can reach $\pm \infty$; if $m_{\theta}$ can reach the value zero, the continuous spectrum will have no gaps, and the spectral model is very similar to that of Pietenpol ${ }^{41}$; if $m_{\theta}$ is bounded away from zero, there will be a gap. Furthermore, the discrete state may occur embedded in either branch of the continuum or may occur within the gap. There are therefore a large variety of covariant models which may be extracted from the framework given here.

Denoting, for each $p$, the second term on the right of (4.1) by

$$
\bar{K}_{p}=\int w(k)|k\rangle\langle k| d^{4} k
$$

a calculation of the type given above, from (1.28) to (1.35), results directly in the relation (2.23) for the reduced resolvent at each $p$.

Somwhat more abstractly, introducing a continuous label $\lambda$, we may denote the continuum generalized eigenstates by $\{|\lambda\rangle\}$, so that (4.3) takes the form

$$
\bar{K}_{p}=\int w(\lambda)|\lambda\rangle\langle\lambda| d \lambda
$$

The reduced resolvent then takes the generalized Friedrichs form

$$
h(z, p)=z-\mathcal{M}_{V}(p)-\int d \lambda \frac{\left|\left\langle\lambda\left|K_{I}\right| \phi_{p}\right)\right|^{2}}{z-w(\lambda)} .
$$

The complex poles which dominate the decay law (in "proper time"), as discussed at the end of Section 2, can then be investigated in a way similar to the discussion of the nonrelativistic form in Section 1. The construction of the generalized states ${ }^{4}$ of the relativistic Lee-Friedrichs model can then be carried out.

We remark here that the generalized eigenstate in this construction corresponds to a shift is the position of the unperturbed eigenvalue of $K_{p}$ from $\mathcal{M}_{V}(p)$ to the complex zero of $h(p, z)$ in the second Riemann sheet. This can be interpreted as the acquisition of a complex part to the total energy-momentum of the system $p^{\mu}$. Let us label the pole position by (we neglect the dependence of $M^{\prime}, \gamma$ on $p$ here)

$$
\zeta=-\frac{M^{\prime 2}}{2 M_{V}}-i \frac{\gamma}{2}
$$

so that, as for the nonrelativistic case, the decay law goes as $e^{-\gamma \tau}$. Then, in the center of momentum frame,

$$
\frac{p^{2}}{2 M_{V}} \rightarrow-\frac{E^{2}}{2 M_{V}} \sim \zeta
$$


or, for $\gamma \ll M^{\prime}$,

$$
E \sim M^{\prime}+i \frac{M_{V}}{2 M^{\prime}} \gamma
$$

For $M^{\prime}=M_{V}+\delta M_{V}$ (we assume $\frac{p^{2}}{2 M_{V}} \sim-\frac{M_{V}}{2}$ for the unperturbed system),

$$
E \sim M_{V}+\delta M_{V}+i \frac{\gamma}{2}
$$

where we have neglected a term of order $\gamma\left(\delta M_{V} / M_{V}\right)$. The result (4.7) illustrates how, in the rest frame of the initial particle, the mass change and decay width generated by the interaction in this covariant model can emerge as a complex energy.

Acknowledgements I would like to thank I. Antoniou and S. Tasaki for discussions, and I. Prigogine for the opportunity to present this work at a seminar at the International Solvay Institutes for Physics and Chemistry at the Free University of Brussels. I am also grateful to N. Shnerb, E. Eisenberg, at Bar Ilan University, L. Burakovsky and M. Land at Tel Aviv University and M. Berkooz, at Rutgers University for helpful discussions, and to W.C. Schieve for his hospitality at the University of Texas at Austin, and for very helpful discussions at an early stage of this work. 


\section{References.}

1. T.D. Lee, Phys. Rev 95, 1329 (1954).

2. K.O. Friedrichs, Comm. Pure and Applied Math. 1, 361 (1950).

3. L.P. Horwitz and J.-P. Marchand, Rocky Mountain Journal of Mathematics 1, 225 (1971).

4. I. Sigal and L.P. Horwitz, Helv. Phys. Acta 51685 (1978); W. Baumgartel, Math. Nachr. 75, 133 (1978); G. Parravicini, V. Gorini, and E.C.G. Sudarshan, Jour. Math. Phys. 21, 2208 (1980); T. Bailey and W.C. Schieve, Nuovo Cimento 47A, 231 (1978); Physics 78, Berlin (1978); A. Bohm, Quantum Mechanics: Foundations and Applications, Springer, Berlin (1986); A Bohm, M. Gadella and G.B. Mainland, Am. Jour. Phys. 57, 1103 (1989).

5. A. Bohm, The Rigged Hilbert Space and Quantum Mechanics, Springer Lecture Notes on Physics 78, Berlin (1978); I.M. Gel'fand and N. Ya. Vilenkin, Generalized Functions, vol 4, Academic Press, N.Y. (1964).

6. I. Antoniou and S. Tasaki, J. Phys. A: Math. Gen 26, 73 (1993); H.H. Hasegawa and W.C. Saphir, Phys. Lett. 161A, 471 (1992); Phys. Rev. A 46, 7401 (1992).

7. I. Antoniou and I. Prigogine, Physica A 192, 443 (1993), and references therein.

8. L.P. Horwitz and C. Piron, Hev. Phys. Acta 46, 316 (1973); R. Fanchi, Phys. Rev D 20, 3108 (1979); C. Dewdney, P.R. Holland, A. Kyprianides and J.P. Vigier, Phys. Lett. A 113, 359 (1986); Phys. Lett. A 114, 444 (1986); A. Kyprianides, Phys. Rep. 155, 1 (1986). 3108 (1979).

9. E.C.G. Stueckelberg, Helv. Phys. Acta 14, 322, 588 (1941); J. Schwinger, Phys. Rev. 82, 664 (1951); R.P. Feynman, Rev. Mod. Phys. 20367 (1948); Phys. Rev. 80, 440 (1950).

10. L.P. Horwitz, Found. of Phys. 22, 421 (1992).

11. See R. Arshansky and L.P. Horwitz, Found. of Phys. 15, 701 (1985) for a discussion of localization in space and time, and the associated Newton-Wigner and LandauPeierls operators. M. Usher and L.P. Horwitz, Found. Phys. Lett. 4, 289 (1991), have discussed the causal properties from the point of view taken by G.C. Hegerfeldt, Phys. Rev. D 10, 3320 (1974); Phys. Rev. Lett. 54, 2395 (1985); Nucl. Phys. B 6, 231 (1989).

12. L.P. Horwitz, W.C. Schieve and C. Piron, Ann. Phys. 137, 306 (1981).

13. L.P. Horwitz, S. Shashoua and W.C. Schieve, Physica A 161, 300 (1989).

14. L. Burakovsky and L.P. Horwitz, Physica A 201, 666 (1993); "Galilean limit of equilibrium relativistic mass distributions," TAUP 2081-93, to be published;" Equilibrium relativistic mass distributions for indistinguishable events," TAUP 2115-93; L. Burakovsky and L.P. Horwitz, "Independence of specific heat on mass distribution for large c," TAUP 2141-94.

15. L. Burakovsky, L.P. Horwitz and W.C. Schieve, "Statistical mechanics of relativistic degenerate Fermi gas I. Cold adiabatic equation of state," TAUP 2136-94.

16. L. Van Hove, Physica 21, 901 (1955); 22, 343 (1956); 23, 441 (1957).

17. R.I. Arshansky and L.P. Horwitz, Jour. Math. Phys. 30, 66, 380 and 213 (1989).See also R. Arshansky and L.P. Horwitz, Phys. Rev. D 29, 2860 (1984).

18. M.C. Land, R.I. Arshansky and L.P. Horwitz, to be published, Found.of Phys. 
19. M.C. Land and L.P. Horwitz, "The Zeeman effect for the relativistic bound state," TAUP-2150-94, to be published.

20. L.P. Horwitz, Jour. Math. Phys. 34, 645 (1993).

21. R. Faibish and L.P. Horwitz, "Non-compact dynamical groups for the solution of bound state problems in relativistic quantum theory I: The relativistic harmonic oscillator in $1+1$ dimensions," TAUP 2025-93, to be published in Jour. Group Theory and Applications; "Dyanmical groups of the relativistic Kepler problem and the harmonic oscillator," vol. II, p. 450, Anales de Fisica, Monografias, vols. I, II, ed. M.A. del Olmo, M. Santander and J.M. Guilarte, CIEMAT-RSEF, Madrid (1993),

22. E.P. Wigner and V.F. Weisskopf, Zeits. f. Phys., 63, 54 (1930); 65, 18 (1930).

23. P. Exner, Open Quantum Systems and Feynman Integrals, D. Reidel, Boston (1985); A. Bohm, Quantum Mechanics: Foundations and Applications, Springer, Berlin (1986). See also L.A. Khalfin, Dokl. Akad. Nauk. USSR, 115, 277 (1957), for early studies of the long and short time behavior.

24. L.P. Horwitz and C. Piron, Helv. Phys. Acta 66, 693 (1993).

25. See, for example, J.-P. Marchand and L.P. Horwitz, Rocky Mountain Jour. Math. 1, 225 (1971)

26. N. Bleistein, R. Handelsman, L.P. Horwitz and H. Neumann, Nuovo Cimento 41A, 389 (1977).

27. E. Henley and W. Thirring, Elementary Quantum Field Theory, McGraw Hill, New York (1963).

28. B. Zumino, in Lectures on Field Theory and the Many Body Problem, ed. E.R. Caianiello, p. 37, Academic Press, New York (1961).

29. L. Van Hove, second of refs. 16.

30. D. Saad, L.P. Horwitz and R.I. Arshansky, Found. Phys. 19, 1126 (1989); N. Shnerb and L.P. Horwitz, Phys. Rev A 48, 4068 (1993).

31. J. Frastai, in preparation.

32. J. Schwinger, Phys. Rev. 82, 664 (1951).

33. W. Pauli and F. Villars, Rev. Mod. Phys. 21, 434 (1949).

34. See, for example, J.M. Jauch and F. Rohrlich, The Theory of Photons and Electrons, 2nd ed., Springer, New York (1976); C. Itzykson and J.-B. Zuber, Quantum Field Theory, McGraw Hill, New York (1980).

35. See, for example, L. de Broglie, Les incertitudes d'Heisenberg et l'interprétation probabiliste de la mécanique ondulatoire, preface by Georges Lochak, Gauthier-Villars, Paris (1982).

36. K. Haller and R.B. Sohn, Phys. Rev A 20, 1541 (1979); K. Haller, Acta Phys. Austriaca 42, 163 (1975); K. Haller, Phys. Rev D 36, 1830(1987).

37. L.P. Horwitz, C. Piron and F. Reuse, Helv. Phys. Acta 48, 546 (1975); C. Piron and F. Reuse, Helv. Phys. Acta 51, 146 (1978);L.P. Horwitz and R. Arshansky, J. Phys. A: Math. Gen. 15, L659 (1982); A. Arensburg and L.P. Horwitz, Found. Phys. 22, 1025 (1992).

38. P.A.M. Dirac, The Principles of Quantum Mechanics, 3rd ed., Oxford University Press, London (1947).

39. G. Källén and W. Pauli, Mat. Fys. Medd. Dan. Vid. Selsk. 30, 1 (1955). 
40. L.P. Horwitz and Y. Lavie, Phys. Rev D 26, 819 (1982).

41. J.L. Pietenpol, Phys. Rev. 162, 1301 (1967); I. Antoniou, J. Levitan and L.P. Horwitz, J. Phys. A: Math.Gen. 26, 6033 (1993). 


\section{Figure Captions}

fig. 1. The process $N \rightarrow V+\theta\left(k^{0}>0\right)$

fig. 2. The process $N \rightarrow V+\theta\left(k^{0}<0\right)$

fig. 3. The process $N+\bar{\theta}\left(k^{0}>0\right) \rightarrow V$ (virtual) 
This figure "fig1-1.png" is available in "png" format from: http://arxiv.org/ps/hep-th/9404154v1 
This figure "fig1-2.png" is available in "png" format from: http://arxiv.org/ps/hep-th/9404154v1 
This figure "fig1-3.png" is available in "png" format from: http://arxiv.org/ps/hep-th/9404154v1 